\title{
Scaling parameters in anomalous and nonlinear Hall effects depend on temperature
}

\author{
Cong Xiao, Hailong Zhou, and Qian Niu \\ Department of Physics, The University of Texas at Austin, Austin, Texas 78712, USA
}

\begin{abstract}
In the study of the anomalous Hall effect, the scaling relations between the anomalous Hall and longitudinal resistivities play the central role. The scaling parameters by definition are fixed as the scaling variable (longitudinal resistivity) changes. Contrary to this paradigm, we unveil that the electron-phonon scattering can result in apparent temperature-dependence of scaling parameters when the longitudinal resistivity is tuned through temperature. An experimental approach is proposed to observe this hitherto unexpected temperature-dependence. We further show that this phenomenon also exists in the nonlinear Hall effect in nonmagnetic inversion-breaking materials and may help identify experimentally the presence of the side-jump contribution besides the Berry-curvature dipole.
\end{abstract}

The anomalous Hall effect [1] has been a fruitful topic of condensed matter physics, providing a paradigm widely-employed to understand related nonequilibrium phenomena such as spin and valley Hall effects [2, 3, and spin-orbit torque [4. In time-reversal broken multiband electronic systems with strong spin-orbit coupling, the anomalous Hall effect originates from both the momentum-space Berry curvature and scattering off disorder [5 7]. In experiments the scaling relations linking the anomalous Hall resistivity $\rho_{\mathrm{AH}}$ to the longitudinal resistivity $\rho$ play the central role in identifying various contributions 8 - 21 .

The well-established theory taking into account a given type of weak-potential static impurities [1, 6, 22, 23, results in the scaling relation

$$
-\rho_{\mathrm{AH}, 0}=\alpha_{0} \rho_{0}+\left(c+c_{0}+c_{00}\right) \rho_{0}^{2} .
$$

Henceforth the subscripts "0" and "1" represent contributions from electron-impurity and electron-phonon scattering, respectively. In this scaling $\alpha_{0}$ arises from the skew scattering 24, $c$ is the Berry-curvature contribution, and $c_{0}$ results from scattering-induced coordinateshift 25, namely the side-jump [5, 26..$c_{00}$ incorporates scattering-induced contributions that are not related to coordinate-shift but share the same scaling behavior as the side-jump one [5, 23], and thereby is referred to as the side-jump-like contribution [27. $\alpha_{0}, c, c_{0}$ and $c_{00}$ do not depend on the density of scatterers thus serve as scaling parameters, and $\rho_{0}$ tuned via changing the density of scatterers plays the role of a scaling variable.

On the other hand, in many experiments the resistivity is tuned through temperature $(T)$ in a wide range where the electron-phonon scattering is important [8, 9, 11-13, 16-19]. For this scattering, most previous theoretical and experimental researches suggest the scaling relation 8, 9, 17, 26, 28, 29.

$$
-\rho_{\mathrm{AH}, 1}=\left(c+c_{1}+c_{11}\right) \rho_{1}^{2},
$$

where the scaling parameters $c_{1}$ and $c_{11}$ are thought, according to the aforementioned characteristic of side-jump and side-jump-like contributions, to be independent of the density of phonons and thus of $T$ 17, 26, 29.
In the presence of both impurities and phonons, when assuming the Matthiessen's rule $\rho=\sum_{i=0,1} \rho_{i}$, a twovariable scaling based on the above two scalings reads [17. $\left(\rho \gg \rho_{\mathrm{AH}}, \sigma_{\mathrm{AH}} \simeq-\rho_{\mathrm{AH}} / \rho^{2}\right)$

$$
\sigma_{\mathrm{AH}}=\alpha_{0} \rho_{0} / \rho^{2}+c+\sum_{i=0,1} c_{i} \rho_{i} / \rho+\sum_{i, j=0,1} c_{i j} \rho_{i} \rho_{j} / \rho^{2} .
$$

Here $c_{10}+c_{01}$ represents the combined effect of scatterings off impurities and phonons, and is also regarded to remain constant as $T$ changes in previous studies [17 21].

In this Letter we uncover that the above widelyaccepted paradigm misses the physics that $c_{1}, c_{11}$ and $c_{10}+c_{01}$ can be strongly $T$ dependent as $T$ drops below the high- $T$ classical equipartition regime where $\rho_{1} \propto T$ [30. In the minimal model of the anomalous Hall effect, namely the two-dimensional (2D) massive Dirac model, Eq. (3a) is demonstrated with all c's given explicitly (detailed later) and reorganized into

$$
\sigma_{\mathrm{AH}}-\alpha_{0} \sigma_{x x}^{2} / \sigma_{0}=\beta+\beta^{\prime} \sigma_{x x} / \sigma_{0}+\beta^{\prime \prime}\left(\sigma_{x x} / \sigma_{0}\right)^{2},
$$

where $\sigma_{0}^{-1}=\rho_{0}$. The $T$ dependence of

$$
\begin{aligned}
\beta(T) & =c+c_{1}(T)+c_{11}(T), \\
\beta^{\prime}(T) & =c_{0}-c_{1}(T)+c_{01}(T)+c_{10}(T)-2 c_{11}(T), \\
\beta^{\prime \prime}(T) & =c_{00}+c_{11}(T)-c_{01}(T)-c_{10}(T),
\end{aligned}
$$

are shown in Fig. 1 although they are believed to be $T$ independent in the conventional paradigm of the anomalous Hall effect. Despite that the specific $T$ dependent forms of $\beta$ 's depend on fine details of the model, the revealed possibility of the $T$ dependence of $c_{1}, c_{11}$ and $c_{10}+c_{01}$ is ubiquitous, as shown in the Supplemental Material [31.

This finding indicates that, the conventionally identified "scaling parameters" in Eqs. 22 - (3b) are in fact allowed to vary with temperature when fitting data. From a viewpoint of basic understanding, scaling parameters by definition ought to be fixed as the scaling variable changes, thereby Eqs. (2) - (3b) can not be termed as "scaling relations" when $\rho$ is tuned through temperature. 


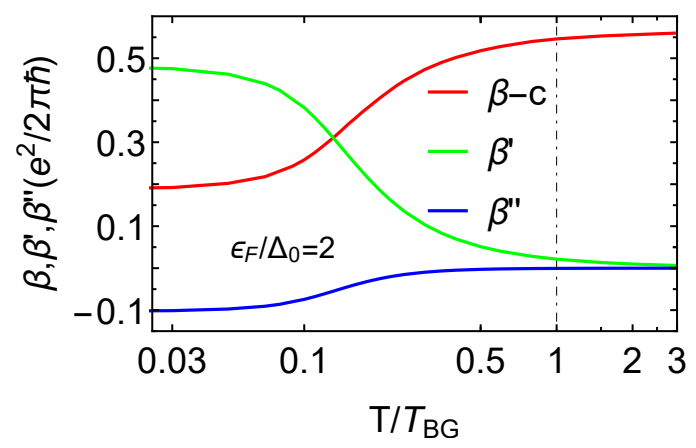

FIG. 1. Temperature-dependence of $\beta$ 's of Eq. $(3 \mathrm{~b})$ in the $2 \mathrm{D}$ massive Dirac model (5) in the presence of both zerorange scalar impurities and acoustic phonons. $T_{B G}$ is the Bloch-Gruneisen temperature [37] which plays the basic role instead of the Debye temperature in 2D metallic systems and marks qualitatively the lower boundary of the high- $T$ classical equipartition regime $\left(T>T_{B G}\right)$ where the resistivity is linear in $T$. When $T$ downs below the equipartition regime the $\beta$ 's become $T$ dependent.

On the practical side, since $\beta, \beta^{\prime}$ and $\beta^{\prime \prime}$ do not depend on the density of impurities $n_{i}$, and thus still serve as scaling parameters when $\rho$ is tuned by changing the density of impurities, we propose an experimental procedure to observe the $T$ dependence of $\beta$ 's.

Now we begin the concrete analysis of the 2D massive Dirac model [5]

$$
\hat{H}_{0}=v\left(\hat{\sigma}_{x} k_{x}+\hat{\sigma}_{y} k_{y}\right)+\Delta_{0} \hat{\sigma}_{z},
$$

where $\hat{\sigma}_{x, y, z}$ are the Pauli matrices, $\mathbf{k}=\left(k_{x}, k_{y}\right)$ is the wave-vector, $v>0$ and $\Delta_{0}>0$ are model parameters. We consider the electron-doped case with Fermi energy $\epsilon_{F}>\Delta_{0}$. For the purpose of revealing the fact that the $\beta$ 's depend on $T$, the quasi-static treatment for acoustic phonons is adequate. This approximation, in which the electron-phonon scattering is treated as an elastic process, produces the correct low- $T$ Bloch-Gruneisen law $\rho_{1} \sim T^{5}$ for three-dimensional (3D) metals [30, 32, 33]. When applied to the side-jump anomalous Hall effect, the high- $T$ and low- $T$ asymptotic behaviors derived within this approximation are the same as those obtained without this approximation [34. Quantitative deviations only appear in the intermediate regime and are not essentially important.

To proceed, we employ the Boltzmann transport theory involving not only on-shell (on the Fermi surface) but also off-shell (away from the Fermi surface) Bloch states [1, 5, 6, 22]. In the presence of scalar quasi-static disorder, the side-jump (sj) and side-jump-like (sjl) contributions to the anomalous Hall conductivity of model (5) are obtained as 31]

$$
\sigma_{\mathrm{AH}}^{\mathrm{sj}}=\frac{e^{2}}{4 \pi \hbar} \sin ^{2} \theta_{F} \cos \theta_{F}\left(\tau_{0}^{-1}-\tau_{1}^{-1}\right) \tau_{t r}
$$

and

$$
\begin{aligned}
\sigma_{\mathrm{AH}}^{\mathrm{sjl}} & =\frac{e^{2}}{64 \pi \hbar} \sin ^{4} \theta_{F} \cos \theta_{F}\left(\tau_{0}^{-1}-\tau_{2}^{-1}\right) \\
& \times\left(3 \tau_{0}^{-1}-4 \tau_{1}^{-1}+\tau_{2}^{-1}\right) \tau_{t r}^{2},
\end{aligned}
$$

respectively. Here $\cos \theta_{F}=\Delta_{0} / \epsilon_{F}, \sin \theta_{F}=v k_{F} / \epsilon_{F}$. $\tau_{t r}^{-1}$ is the value of the inverse transport relaxation time

$$
\tau_{t r}^{-1}(k)=\frac{D_{k}}{\hbar} \int d \phi_{\mathbf{k}^{\prime} \mathbf{k}}\left|\left\langle u_{\mathbf{k}^{\prime}} \mid u_{\mathbf{k}}\right\rangle\right|^{2} W_{\mathbf{k}^{\prime} \mathbf{k}}\left(1-\cos \phi_{\mathbf{k}^{\prime} \mathbf{k}}\right)
$$

on the Fermi surface, and

$$
\tau_{n}^{-1}(k)=\frac{D_{k}}{\hbar} \int d \phi_{\mathbf{k}^{\prime} \mathbf{k}} W_{\mathbf{k}^{\prime} \mathbf{k}} \cos \left(n \phi_{\mathbf{k}^{\prime} \mathbf{k}}\right), \quad n=0,1,2 \ldots
$$

where $D_{k}$ is the density of states, $\phi_{\mathbf{k}^{\prime} \mathbf{k}}$ is angle between $\mathbf{k}$ and $\mathbf{k}^{\prime},\left|u_{\mathbf{k}}\right\rangle$ is the spinor eigenstate in the positive band, and $W_{\mathbf{k}^{\prime} \mathbf{k}}$ is the plane-wave part of the lowest-Born-order scattering rate $\left|\left\langle u_{\mathbf{k}^{\prime}} \mid u_{\mathbf{k}}\right\rangle\right|^{2} W_{\mathbf{k}^{\prime} \mathbf{k}}$.

For quasi-static electron-phonon scattering one has $W_{\mathbf{k}^{\prime} \mathbf{k}}^{\mathrm{ep}}=\frac{2 N_{\mathbf{q}}}{\mathrm{V}}\left|U_{\mathbf{k}^{\prime} \mathbf{k}}^{o}\right|^{2}$, where $U_{\mathbf{k}^{\prime} \mathbf{k}}^{o}$ is the plane-wave part of the electron-phonon matrix element, $\mathrm{V}$ is the volume (area in 2D) of the system, $N_{\mathbf{q}}$ is the Bose occupation function for the phonon model with wave-vector $\mathbf{q}$ and energy $\hbar \omega_{q}$, and the factor 2 accounts for the absorption and emission of phonons [30]. To simplify the analysis we neglect the Umklapp process, thus $\mathbf{q}=\mathbf{k}^{\prime}-\mathbf{k}$. In comparison, for static impurities $W_{\mathbf{k}^{\prime} \mathbf{k}}^{\mathrm{ei}}=n_{i}\left|V_{\mathbf{k}^{\prime} \mathbf{k}}^{o}\right|^{2}$, with $V_{\mathbf{k}^{\prime} \mathbf{k}}^{o}$ the plane-wave part of the matrix element of the impurity potential. Hereafter the superscript "ei/ep" means that the quantity is contributed by the electronimpurity/-phonon scattering alone.

To obtain analytic results, we assume zero-range scalar impurities $\left(\left|V_{\mathbf{k}^{\prime} \mathbf{k}}^{o}\right|^{2}=V_{i}^{2}\right.$ is a constant), isotropic Debye phonons, and the deformation-potential coupling for which a so-called electron-phonon coupling constant $\lambda^{2}=2 \mathrm{~V}^{-1}\left|U_{\mathbf{k}^{\prime} \mathbf{k}}^{o}\right|^{2} / \hbar \omega_{q}$ exists [35, 36]. Then

$$
W_{\mathbf{k}^{\prime} \mathbf{k}}=W_{\mathbf{k}^{\prime} \mathbf{k}}^{\mathrm{ei}}+W_{\mathbf{k}^{\prime} \mathbf{k}}^{\mathrm{ep}}=n_{i} V_{i}^{2}+\lambda^{2} k_{B} T \frac{z}{e^{z}-1},
$$

where $z=\hbar \omega_{q} / k_{B} T=\frac{q}{2 k_{F}} \frac{T_{B G}}{T}$, and $T_{B G}=\hbar c_{s} 2 k_{F} / k_{B}$ ( $c_{s}$ is the sound velocity) is the Bloch-Gruneisen temperature 37. Model results of $\sigma_{\mathrm{AH}}^{\mathrm{SJ}} \equiv \sigma_{\mathrm{AH}}^{\mathrm{sj}}+\sigma_{\mathrm{AH}}^{\mathrm{sjl}}$ are obtained 31] according to

$$
\tau_{n}^{-1} \tau_{t r}=\frac{\left(\tau_{n}^{\mathrm{ei}}\right)^{-1}+\left(\tau_{n}^{\mathrm{ep}}\right)^{-1}}{\left(\tau_{t r}^{\mathrm{ep}}\right)^{-1}+\left(\tau_{t r}^{\mathrm{ep}}\right)^{-1}}, \quad n=0,1,2 \ldots
$$

Next we take into account the skew scattering (sk) from the third-order non-Gaussian impurity correlator $n_{i} V_{i}^{3}$ of zero-range scalar impurities [5, 38]:

$$
\sigma_{\mathrm{AH}}^{\mathrm{sk}}=\frac{e^{2}}{16 \pi \hbar} \sin ^{4} \theta_{F}\left(\frac{\tau_{t r}}{\tau_{0}^{\mathrm{ei}}}\right)^{2} D_{F} V_{i} \frac{\Delta_{0} \tau_{0}^{\mathrm{ei}}}{\hbar},
$$


where $D_{F}$ is the density of states on the Fermi surface.

The model result thus takes the form of

$$
\sigma_{\mathrm{AH}}-c-\sigma_{\mathrm{AH}}^{\mathrm{sk}}=\sum_{n} a_{n}^{\mathrm{sj}} \tau_{n}^{-1} \tau_{t r}+\sum_{n n^{\prime}} b_{n n^{\prime}}^{\mathrm{sjl}} \tau_{n}^{-1} \tau_{n^{\prime}}^{-1} \tau_{t r}^{2},
$$

where $a_{n}^{\mathrm{sj}}$ and $b_{n n^{\prime}}^{\mathrm{sjl}}$ are readable from Eqs. (6) and (7). By noting that $\rho_{0(1)} / \rho=\tau_{t r} / \tau_{t r}^{\mathrm{ei}(\mathrm{ep})}$ and $\sigma_{\mathrm{AH}}^{\mathrm{sk}}=\alpha_{0} \rho_{0} / \rho^{2}$, the above equation can be cast into Eq. (3a), where the impurity-determined coefficients

$$
c_{0}=\sigma_{\mathrm{AH}}^{\mathrm{sj}, \mathrm{ei}}=\sum_{n} a_{n}^{\mathrm{sj}} \frac{\tau_{t r}^{\mathrm{ei}}}{\tau_{n}^{\mathrm{ei}}}, \quad c_{00}=\sigma_{\mathrm{AH}}^{\mathrm{sjl}, \mathrm{ei}}=\sum_{n n^{\prime}} b_{n n^{\prime}}^{\mathrm{sjl}} \frac{\left(\tau_{t r}^{\mathrm{ei}}\right)^{2}}{\tau_{n}^{\mathrm{ei}} \tau_{n^{\prime}}^{\mathrm{ei}}},
$$

and $\alpha_{0} \sim D_{F} V_{i} \tau_{t r}^{\text {ei }} / \tau_{0}^{\text {ei }}$ are independent of the density of impurities, while the phonon-determined coefficients, namely

$$
c_{1}=\sigma_{\mathrm{AH}}^{\mathrm{sj}, \mathrm{ep}}=\sum_{n} a_{n}^{\mathrm{sj}} \frac{\tau_{t r}^{\mathrm{ep}}}{\tau_{n}^{\mathrm{ep}}}, \quad c_{11}=\sigma_{\mathrm{AH}}^{\mathrm{sjl}, \mathrm{ep}}=\sum_{n n^{\prime}} b_{n n^{\prime}}^{\mathrm{sjl}} \frac{\left(\tau_{t r}^{\mathrm{ep}}\right)^{2}}{\tau_{n}^{\mathrm{ep}} \tau_{n^{\prime}}^{\mathrm{ep}}},
$$

are $T$ dependent at low temperatures below the high- $T$ equipartition regime as shown in Fig. 2(a). This implies that Eq. (2) can not be theoretically viewed as a scaling relation, since the conventionally identified "scaling paramter" $c_{1}+c_{11}$ in fact changes as $\rho_{1}$ varies with temperature. Equations (3a) and (3b) suffer from the same situation. Meanwhile, the combined contribution from the impurity and phonon scatterings to the side-jumplike anomalous Hall conductivity

$$
c_{01}+c_{10}=\sum_{n n^{\prime}} b_{n n^{\prime}}^{\mathrm{sjl}}\left(\frac{\tau_{t r}^{\mathrm{ei}}}{\tau_{n}^{\mathrm{ei}}} \frac{\tau_{t r}^{\mathrm{ep}}}{\tau_{n^{\prime}}^{\mathrm{ep}}}+\frac{\tau_{t r}^{\mathrm{ep}}}{\tau_{n}^{\mathrm{ep}}} \frac{\tau_{t r}^{\mathrm{ei}}}{\tau_{n^{\prime}}^{\mathrm{ei}}}\right)
$$

also depends on $T$ below the equipartition regime. The $T$ dependence of $c_{1}, c_{11}$ and $c_{01}+c_{10}$ yields that of the $\beta$ 's shown in Fig. 1 .

The qualitative picture for the appearance of the $T$ dependence is simple: the side-jump and side-jumplike contributions are conventionally viewed as independent of scattering time for a given source of scattering [7, 26, 39, but in general they are just zeroth-order homogeneous terms of scattering time [38, as is apparent in Eqs. (6) and (7). In the equipartition regime the $T$ dependence of electron-phonon scattering times in the denominator and numerator of these zeroth-order homogeneous terms are the same $\left(T^{-1}\right)$, and thereby drop out of $\beta$ 's. While at lower temperatures below the equipartition regime the bosonic nature of phonon occupation number makes the $T$ dependence irreducible even in the zeroth-order homogeneous terms.

The model analysis also offers a perspective to understand why the conventional idea of $T$ independent scaling parameters works practically in tuning- $T$ experiments. Because in the high- $T$ regime $W^{\mathrm{ep}}=\lambda^{2} k_{B} T$ drops out
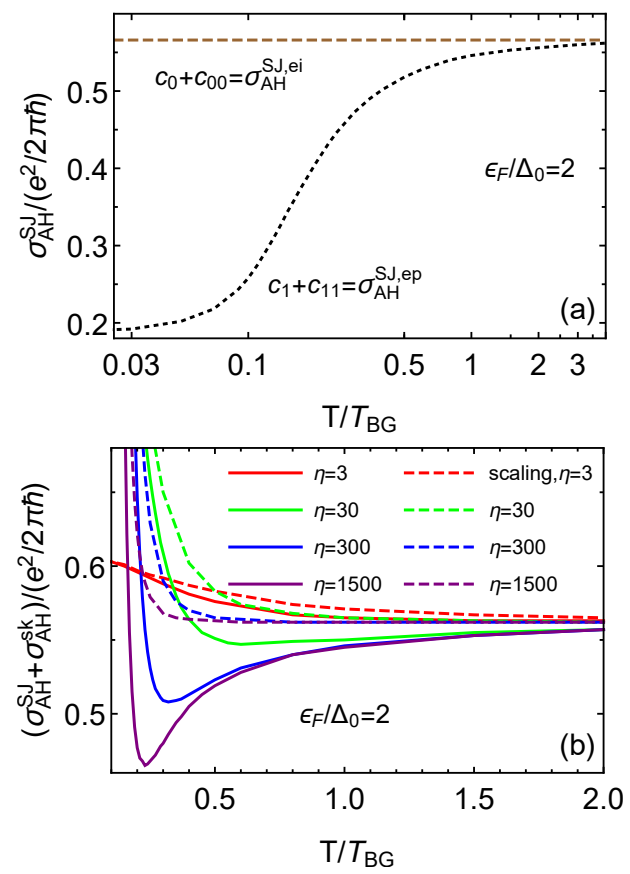

FIG. 2. Temperature-dependence of (a) $\sigma_{\mathrm{AH}}^{\mathrm{SJ}, \text { ei }}$ and $\sigma_{\mathrm{AH}}^{\mathrm{SJ}, \text { ep }}$, and of (b) $\sigma_{\mathrm{AH}}^{\mathrm{SJ}}+\sigma_{\mathrm{AH}}^{\mathrm{sk}}=\sigma_{\mathrm{AH}}-c$ for $\epsilon_{F} / \Delta_{0}=2$ in model $(5)$ in the presence of zero-range scalar impurities and acoustic phonons. Larger values of the parameter $\eta$ correspond to smaller impurity density. The dashed curves in (b) are obtained by assuming the scaling relation 17 in the presence of both scattering sources. In the weak scattering regime $D_{F}\left|V_{i}\right| \ll 1$ and $\Delta_{0} \tau_{0}^{\mathrm{ei}} / \hbar \gg 1$. Thus in the calculation of $\sigma_{\mathrm{AH}}^{\mathrm{sk}}$ we take $D_{F} V_{i}=10^{-3}, \Delta_{0} \tau_{0}^{\mathrm{ei}} / \hbar=10^{j+2}$ for $\eta=3 \times 10^{j}(j=0,1,2)$, and $\Delta_{0} \tau_{0}^{\mathrm{ei}} / \hbar=5 \times 10^{4}$ for $\eta=1500$ ( $n_{i}$ is tuned).

of $\tau_{t r} / \tau_{n}$, the value of $\sigma_{\mathrm{AH}}^{\mathrm{SJ}}$ contributed by phonons coincides with that contributed by zero-range scalar impurities. This value also applies in the presence of both scattering sources since $W=n_{i} V_{i}^{2}+\lambda^{2} k_{B} T$ for this case also drops out of $\tau_{t r} / \tau_{n}$. Therefore, in the high- $T$ regime the scalar zero-range impurities and acoustic phonons are indistinguishable in inducing $\sigma_{\mathrm{AH}}^{\mathrm{SJ}}$, namely, $c_{1}=c_{0}$ and $c_{i j}=c_{00}$. Then, if the electron-phonon scattering related $c$ 's took $T$ independent values, just following the conventional idea, the scaling relation would hold and read

$$
\sigma_{\mathrm{AH}}=\alpha_{0} \sigma_{0}^{-1} \sigma_{x x}^{2}+c+c_{0}+c_{00}
$$

As is shown by the dashed curves in Fig. 2(b), this scaling relation well describes the $T$ dependence of the anomalous Hall conductivity in moderately dirty systems with smaller $\eta$. Here the dimensionless parameter $\eta=\lambda^{2} k_{B} T_{B G} / n_{i} V_{i}^{2}$ is introduced to denote the purity of system: larger $\eta$ means smaller $n_{i}$. In our model case $T_{B G}$ is the lower boundary $T_{L}$ of the equipartition regime where $\rho_{1} \propto T$, thus $\eta \simeq \tau_{0}^{\mathrm{ei}} / \tau_{0}^{\mathrm{ep}}\left(T_{L}\right)=\tau_{t r}^{\mathrm{ei}} / \tau_{t r}^{\mathrm{ep}}\left(T_{L}\right)$, tak- 
ing the instructive form of

$$
\eta \simeq \frac{\rho_{1}\left(T_{L}\right)}{\rho_{0}}=\frac{\rho\left(T_{L}\right)-\rho_{0}}{\rho_{0}},
$$

which can be read out conveniently from transport experiments. Given the smaller $\eta$ (usually $<2$ ) of the samples used in previous tuning- $T$ experiments, the above argument provides a clue to understand the approximate validity of scaling relations in these experiments.

As is shown by Fig. 2(b), the deviation of $\sigma_{\mathrm{AH}}$ caused by assuming the scaling relation is apparent only in highpurity systems. By contrast, it is worthwhile to emphasize that in Eq. (3b) the $\beta$ 's do not depend on $\rho_{0}$, thus their $T$ dependence is anticipated to show up irrespective of the sample quality.

Next we propose an experimental procedure to observe the predicted $T$ dependence of $\beta$ 's, based on the recently developed thin film approach in the study of the anomalous Hall effect [16 18. In this approach the effective impurity density can be continuously manipulated by tuning the thickness of single crystalline magnetic thin films (the Curie temperature is assumed to be much higher than the Debye temperature, which is the case of $\mathrm{Fe}$ and $\mathrm{Co}$ ), meanwhile the electronic band structure does not change in the thickness range. In the low- $T$ limit $\sigma_{x x}=\sigma_{0}$, and Eq. (3b) reduces to the linear scaling $\sigma_{\mathrm{AH}}=\alpha_{0} \sigma_{0}+c+c_{0}+c_{00}$, thereby $\alpha_{0}$ can be extracted by tuning $\sigma_{0}$ through the film thickness. Because in Eq. (3b the $\beta$ 's are still scaling parameters that remain unchanged when the film thickness is tuned, it is reasonable to plot $\left(\sigma_{\mathrm{AH}}-\alpha_{0} \sigma_{0}^{-1} \sigma_{x x}^{2}\right)$ versus $\sigma_{0}^{-1} \sigma_{x x}$ through tuning the film thickness for every chosen fixed temperature. One can then extract the $\beta$ 's for different temperatures from the high- $T$ equipartition regime $T>T_{L}$ (experiments in common $3 \mathrm{D}$ metals often show $T_{L} \simeq T_{D} / 3$ as the lower boundary of the $\rho_{1} \propto T$ regime, with $T_{D}$ the Debye temperature [40, 41]) down to the low- $T$ residual-resistivity regime. The $T$-variation curves of $\beta$ 's are thus obtained. The predicted $T$ independent values of $\beta$ 's at $T>T_{L}$ can be determined first, whereas their $T$-dependence can be observed as temperature downs below $T_{L}$.

Finally we extend the discussion to the nonlinear Hall effect - a second-order Hall current response to the electric field $E_{x}$ in nonmagnetic systems with inversion breaking [42 51]: $j_{y}=\chi_{y x x} E_{x} E_{x}$, with $\chi_{y x x}$ the response coefficient. The systematic Boltzmann analysis of $\chi_{y x x}$, which is of the linear order of scattering time, incorporates the Berry-curvature dipole (bcd) mechanism 42 and the nonlinear generalizations of the side-jump, side-jump-like and skew scattering contributions [52 [57]. Naturally, equation (3a) has been extended to the nonlinear Hall effect in the dc limit as 55

$$
\frac{V_{y}^{N}}{\left(V_{x}^{L}\right)^{2}}=C+A_{0} \frac{\rho_{0}}{\rho_{x x}^{2}}+\sum_{i=0,1} C_{i} \frac{\rho_{i}}{\rho_{x x}}+\sum_{i, j=0,1} C_{i j} \frac{\rho_{i} \rho_{j}}{\rho_{x x}^{2}}
$$

where $V_{y}^{N}$ and $V_{x}^{L}$ are the nonlinear Hall and linear longitudinal voltage, respectively, and $V_{y}^{N} /\left(V_{x}^{L}\right)^{2}=\chi_{y x x} \rho_{x x}$. Here the notation $\rho_{x x}$ is used instead of $\rho$, considering the low-symmetry of the materials for observing the nonlinear Hall effect [44, 45]. Equivalently,

$V_{y}^{N} /\left(V_{x}^{L}\right)^{2}-A_{0} \sigma_{x x}^{2} / \sigma_{0}=B+B^{\prime} \sigma_{x x} / \sigma_{0}+B^{\prime \prime}\left(\sigma_{x x} / \sigma_{0}\right)^{2}$,

where $B=C+C_{1}+C_{11}, B^{\prime}=C_{0}-C_{1}+C_{01}+C_{10}-2 C_{11}$ and $B^{\prime \prime}=C_{00}+C_{11}-C_{01}-C_{10}$. Here all $C^{\prime}$ 's are zeroth-order homogeneous terms of scattering time [55]. In particular, $C=\chi_{y x x}^{\mathrm{bcd}} \rho_{x x}, C_{0(1)}=\chi_{y x x}^{\text {sj,ei(ep) }} \rho_{0(1)}$ and $C_{00(11)}=\chi_{y x x}^{\text {sjl,ei(ep) }} \rho_{0(1)}$. Following the conventional paradigm of the anomalous Hall effect [17, 18, in the previous understanding Eq. 20 is viewed as a scaling relation when tuning temperature, and the $B$ 's are believed to be $T$ independent [55].

According to our work on the anomalous Hall effect, however, it is apparent that the $B$ 's are in fact $T$ dependent. An experimental procedure similar to the aforementioned one for the anomalous Hall effect can be applied to verify this idea. The $T$ dependence of $C$ resulted from the Berry-curvature dipole can be regarded to be weak 44, as is verified in the relaxation time approximation [55], under which $C$ is independent of scattering time. Thus the $T$ dependence of the $B$ 's arises from that of the phonon-related side-jump and side-jump-like zeroth-order homogeneous terms, e.g., $C_{1}$ and $C_{11}$. In the high- $T$ regime where $\rho_{1} \propto T$, the $T$ dependence of electron-phonon scattering times in the denominator and numerator of these terms are the same $(1 / T)$, and thereby drop out, leading to $T$ independent $B$ 's. While at lower temperatures, the bosonic phonon-occupation leaves the $T$ dependence irreducible. Illustration of this argument using a prototypical model of the nonlinear Hall effect, namely the 2D tilted massive Dirac model [42, 53, 55, 56, is presented in the Supplemental Material 31 .

In a recent experiment on the nonlinear Hall effect in bilayer $\mathrm{WTe}_{2}$ 45, only the Berry-curvature dipole mechanism was claimed. Whereas in another experiment on few-layer $\mathrm{WTe}_{2}$ [4], a scaling taking the form of (17) $\left(\sigma_{\mathrm{AH}} \rightarrow V_{y}^{N} /\left(V_{x}^{L}\right)^{2}\right)$ was observed in tuning- $T$ measurements, indicating the presence of the skew scattering. Since the experimental system is dirty $(\eta<1)$, the emergence of scaling (17) in practice is reasonable. However, the tuning- $T$ measurement alone can not distinguish the side-jump (-like) contribution from the Berry-curvature dipole. In order to further investigate the relevance of the side-jump (-like) contribution, a possible route suggested by our results is to observe the $T$ dependence of $B$ in Eq. 20 by using multi-step $\mathrm{WTe}_{2}$ samples through the above described experimental approach.

In summary, we have uncovered that, the conventionally identified scaling parameters, which play the central 
role in the study of the anomalous Hall effect, in fact depend on temperature below the equipartition regime. An experimental approach has been proposed to observe this hitherto unexpected temperature-dependence. We also showed that the similar physics applies to the recently proposed scaling relations for the nonlinear Hall effect, and provides a possible approach to identifying experimentally the relevance of the side-jump contribution besides the Berry-curvature dipole.

We thank Ming Xie, Shengyuan A. Yang, Zongzheng Du, Haizhou Lu, Dazhi Hou and Tingxin Li for insightful discussions. We are especially indebted to Zongzheng $\mathrm{Du}$ and Haizhou Lu for sharing their unpublished supplementary material. Q.N. is supported by DOE (DEFG03-02ER45958, Division of Materials Science and Engineering) on the model analysis in this work. C.X. and H.Z. are supported by NSF (EFMA-1641101) and Welch Foundation (F-1255).

[1] N. Nagaosa, J. Sinova, S. Onoda, A. H. MacDonald, and N. P. Ong, Rev. Mod. Phys. 82, 1539 (2010).

[2] J. Sinova, S. O. Valenzuela, J. Wunderlich, C. H. Back, and T. Jungwirth, Rev. Mod. Phys. 87, 1213 (2015).

[3] D. Xiao, W. Yao, and Q. Niu, Phys. Rev. Lett. 99, 236809 (2007).

[4] C. Xiao and Q. Niu, Phys. Rev. B 96, 045428 (2017).

[5] N. A. Sinitsyn, A. H. MacDonald, T. Jungwirth, V. K. Dugaev, and J. Sinova, Phys. Rev. B 75, 045315 (2007).

[6] N. A. Sinitsyn, J. Phys.: Condens. Matter 20, 023201 (2008).

[7] A. A. Kovalev, J. Sinova, and Y. Tserkovnyak, Phys. Rev. Lett. 105, 036601 (2010).

[8] P. N. Dheer, Phys. Rev. 156, 637 (1967).

[9] R. W. Klaffky and R. V. Coleman, Phys. Rev. B 10, 2915 (1974).

[10] W.-L. Lee, S. Watauchi, V. L. Miller, R. J. Cava, and N. P. Ong, Phys. Rev. Lett. 93, 226601 (2004).

[11] S. H. Chun, Y. S. Kim, H. K. Choi, I. T. Jeong, W. O. Lee, K. S. Suh, Y. S. Oh, K. H. Kim, Z. G. Khim, J. C. Woo, and Y. D. Park, Phys. Rev. Lett. 98, 026601 (2007).

[12] T. Miyasato, N. Abe, T. Fujii, A. Asamitsu, S. Onoda, Y. Onose, N. Nagaosa, and Y. Tokura, Phys. Rev. Lett. 99, 086602 (2007).

[13] Y. Pu, D. Chiba, F. Matsukura, H. Ohno, and J. Shi, Phys. Rev. Lett. 101, 117208 (2008).

[14] K. M. Seemann, Y. Mokrousov, A. Aziz, J. Miguel, F. Kronast, W. Kuch, M. G. Blamire, A. T. Hindmarch, B. J. Hickey, I. Souza, and C. H. Marrows, Phys. Rev. Lett. 104, 076402 (2010).

[15] P. He, L. Ma, Z. Shi, G. Y. Guo, J. G. Zheng, Y. Xin, and S. M. Zhou, Phys. Rev. Lett. 109, 066402 (2012).

[16] Y. Tian, L. Ye, and X. Jin, Phys. Rev. Lett. 103, 087206 (2009).

[17] D. Hou, G. Su, Y. Tian, X. Jin, S. A. Yang, and Q. Niu, Phys. Rev. Lett. 114, 217203 (2015).

[18] D. Yue and X. Jin, J. Phys. Soc. Jpn. 86, 011006 (2017). [19] M. Meng, S. X. Wu, W. Q. Zhou, and S. W. Li, Appl.
Phys. Lett. 109, 082405 (2016).

[20] K. K. Meng, J. Miao, X. G. Xu, J. X. Xiao, J. H. Zhao, and Y. Jiang, Phys. Rev. B 93, 060406(R) (2016).

[21] Y. Omori, E. Sagasta, Y. Niimi, M. Gradhand, L. E. Hueso, F. Casanova, and Y. C. Otani, Phys. Rev. B 99, 014403 (2019).

[22] W. Kohn and J. M. Luttinger, Phys. Rev. 108, 590 (1957); J. M. Luttinger, Phys. Rev. 112, 739 (1958).

[23] I. A. Ado, I. A. Dmitriev, P. M. Ostrovsky, and M. Titov, Phys. Rev. B 96, 235148 (2017).

[24] J. Smit, Physica (Amsterdam) 21, 877 (1955); 24, 39 (1958).

[25] N. A. Sinitsyn, Q. Niu, and A. H. MacDonald, Phys. Rev. B 73, 075318 (2006).

[26] L. Berger, Phys. Rev. B 2, 4559 (1970).

[27] In the review paper [1] $c_{00}$ is also included into the category of side-jump, whereas in some other papers (e.g., Refs. 6, 23] ) $c_{00}$ is not called side-jump. The present terminology can be viewed as a compromise between different opinions in the community.

[28] H. R. Leribaux, Phys. Rev. 150, 384 (1966).

[29] A. Crepieux and P. Bruno, Phys. Rev. B 64, 014416 (2001).

[30] J. M. Ziman, Principles of the Theory of Solids (Cambridge University Press, Cambridge, 1972).

[31] See Supplemental Material for calculation details of Figs. 1 and 2, the derivation of Eqs. (6) and (7), modelindependent analysis of the $T$ dependence of $\beta$ 's in the anomalous Hall effect, and analysis of the $T$ dependence of $B$ 's in the nonlinear Hall effect of the 2D tilted massive Dirac model.

[32] N. W. Ashcroft and N. D. Mermin, Solid State Physics (Saunders, Philadelphia, 1976).

[33] M. Marder, Condensed Matter Physics (John Wiley \& Sons, Inc., New York, 2000).

[34] C. Xiao, Y. Liu, M. Xie, S. A. Yang, and Q. Niu, Phys. Rev. B 99, 245418 (2019).

[35] A. A. Abrikosov, L. P. Gor'kov, and I. E. Dzyaloshinskii, Quantum Field Theoretical Methods in Statistical Physics (Pergamon Press, New York, 1965).

[36] J. Rammer and H. Smith, Rev. Mod. Phys. 58, 323 (1986).

[37] D. K. Efetov and P. Kim, Phys. Rev. Lett. 105, 256805 (2010).

[38] S. A. Yang, H. Pan, Y. Yao, and Q. Niu, Phys. Rev. B 83, 125122 (2011).

[39] J. Weischenberg, F. Freimuth, J. Sinova, S. Blugel, and Y. Mokrousov, Phys. Rev. Lett. 107, 106601 (2011).

[40] J. M. Ziman, Electrons and Phonons (Clarendon, Oxford, 1960).

[41] G. K. White and S. B. Woods, Phil. Trans. Roy. Soc. (London) A251, 273 (1958).

[42] I. Sodemann and L. Fu, Phys. Rev. Lett. 115, 216806 (2015).

[43] T. Low, Y. Jiang, and F. Guinea, Phys. Rev. B 92, 235447 (2015).

[44] K. Kang, T. Li, E. Sohn, J. Shan, and K. F. Mak, Nat. Mater. 18, 324-328 (2019).

[45] Q. Ma, et al., Nature 565, 337-342 (2019).

[46] J.-S. You, S. Fang, S.-Y. Xu, E. Kaxiras, and T. Low, Phys. Rev. B 98, 121109(R) (2018).

[47] Y. Zhang, J. van den Brink, C. Felser, and B. Yan, 2D Materials 5, 044001 (2018).

[48] S.-Y. Xu et al., Nature Physics 14, 900 (2018). 
[49] J. I. Facio, D. Efremov, K. Koepernik, J.-S. You, I. Sodemann, and J. van den Brink, Phys. Rev. Lett. 121, 246403 (2018).

[50] Z. Z. Du, C. M. Wang, H.-Z. Lu, and X. C. Xie, Phys. Rev. Lett. 121, 266601 (2018).

[51] B. T. Zhou, C.-P Zhang, and K. T. Law, arXiv:1903.11958 (2019).

[52] E. Deyo, L. E. Golub, E. L. Ivchenko, and B. Spivak,
arXiv:0904.1917 (2009).

[53] E. J. Konig, M. Dzero, A. Levchenko and D. A. Pesin, Phys. Rev. B 99, 155404 (2019).

[54] H. Isobe, S.-Y. Xu, and L. Fu, arXiv:1812.08162 (2018).

[55] Z. Z. Du, C. M. Wang, H.-Z. Lu, and X. C. Xie, Nat. Commun. 10, 3047 (2019).

[56] S. Nandy and I. Sodemann, arXiv:1901.04467 (2019).

[57] C. Xiao, Z. Z. Du, and Q. Niu, arXiv:1907.00577 (2019). 